\title{
ACE-inhibition, but not weight reduction restores cardiomyocyte response to $\beta$-adrenergic stimulation in the metabolic syndrome
}

Ines Nevelsteen ${ }^{1}$, Virginie Bito ${ }^{2}$, Gerry Van der Mieren ${ }^{1}$, Annelies Vanderper ${ }^{1}$, An Van den Bergh ${ }^{1}$, Karin R Sipido ${ }^{2}$, Kanigula Mubagwa ${ }^{1}$ and Paul Herijgers ${ }^{1 *}$

\begin{abstract}
Background: Diabetic cardiomyopathy is characterized by systolic and early diastolic ventricular dysfunction. In the metabolic syndrome (MS), ventricular stiffness is additionally increased in a later stage. It is unknown whether this is related to intrinsic cardiomyocyte dysfunction, extrinsic factors influencing cardiomyocyte contractility and/or cardiac function, or a combination of both. A first aim was to study cardiomyocyte contractility and $\mathrm{Ca}^{2+}$ handling in vitro in a mouse model of MS. A second aim was to investigate whether in vivo hypocaloric diet or ACE-inhibition (ACE-I) improved cardiomyocyte contractility in vitro, contractile reserve and $\mathrm{Ca}^{2+}$ handling.

Methods: This study was performed in LDL-receptor (LDLR-/-) and leptin-deficient (ob/ob), double knock-out mice (DKO), featuring obesity, type II diabetes, atherogenic dyslipidemia and hypertension. Single knock-out LDLR-/-, ob/ob and wild type mice were used as controls. Cellular contractility, $\mathrm{Ca}^{2+}$ handling and their response to in vivo treatment with diet or ACE-I were studied in isolated cardiomyocytes at baseline, during $\beta$-adrenergic stimulation or increased extracellular $\mathrm{Ca}^{2+}$, using field stimulation and patch-clamp.

Results: In untreated conditions, prolongation of contraction-relaxation cycle and altered $\mathrm{Ca}^{2+}$ handling are observed in MS. Response to increased extracellular $\mathrm{Ca}^{2+}$ and $\beta$-adrenergic stimulation is impaired and could not be rescued by weight loss. ACE-I restored impaired response to $\beta$-adrenergic stimulation in MS, but not the decreased response to increased extracellular $\mathrm{Ca}^{2+}$.
\end{abstract}

Conclusions: Cardiomyocyte contractility and $\beta$-adrenergic response are impaired in MS, due to alterations in cellular $\mathrm{Ca}^{2+}$ handling. ACE-I, but not weight loss, is able to restore cardiomyocyte response to $\beta$-adrenergic stimulation in MS.

Keywords: Metabolic syndrome, Cardiomyocyte contractility, $\beta$-adrenergic stimulation, Hypocaloric diet, ACE-inhibition

\section{Background}

The metabolic syndrome (MS) is characterized by the co-occurrence of at least three of the following features: abdominal obesity, hypertension, dyslipidemia, insulin resistance \pm glucose intolerance, pro-inflammatory and pro-thrombotic status. The prevalence of MS and diabetes mellitus type II (DMII) is increasing. Affected patients are at higher risk of developing cardiovascular complications, caused by diabetic, combined with ischemic,

\footnotetext{
* Correspondence: Paul.herijgers@med.kuleuven.be

'Department of Cardiovascular Sciences, Research Unit of Experimental

Cardiac Surgery, KU Leuven, Herestraat 49, Leuven B-3000, Belgium

Full list of author information is available at the end of the article
}

cardiomyopathy and angiopathy. The existence of a primary diabetic cardiomyopathy has been shown in diabetic animal models [1]. The co-occurrence of hypertension and dyslipidemia in DMII aggravates cardiovascular dysfunction in vivo [2].

MS-associated cardiomyopathy can be distinguished in vivo from diabetic cardiomyopathy by impaired late diastolic function, lower end-diastolic volume, decreased cardiac output and concentric hypertrophy [2].

Cardiac function is determined by intrinsic cardiomyocyte function, but also influenced by myoarchitecture, composition and structure of the extracellular matrix, by preload and afterload. It is unclear whether the in vivo

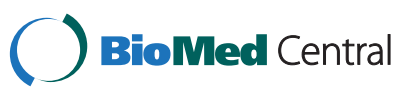


features of heart failure in MS are caused by intrinsic cardiomyocyte dysfunction and/or extrinsic factors influencing cardiomyocyte or cardiac contractility. No studies are available on isolated cardiomyocyte contractility in MS-mice.

Calcium plays a key role in excitation-contraction coupling in cardiomyocyte contractility. Myocyte contraction is determined by entry of $\mathrm{Ca}^{2+}$ via L-type calcium channels that triggers release of $\mathrm{Ca}^{2+}$ from the sarcoplasmatic reticulum (SR) via ryanodine receptors (RyR) and activation of myofilaments. Relaxation occurs with sequestration of $\mathrm{Ca}^{2+}$ from the cytosol to the SR by SR $\mathrm{Ca}^{2+}$ ATP-ase (SERCA2a) and extrusion of $\mathrm{Ca}^{2+}$ out of the cell by the sodium-calcium exchanger (NCX). Alterations in $\mathrm{Ca}^{2+}$ cycling in DMII and failing hearts have been extensively studied [3-5]. We have shown previously in ventricular homogenates of MS-mice that phosphorylation of phospholamban (PLB) is decreased and associated with an increased SERCA2a-expression [2]. Although these data provide insights into contributing mechanisms to contractile dysfunction in vivo, changes in intrinsic properties of cardiomyocytes have not been studied in MS-mice.

Diet and ACE-I are the golden standard in the treatment of DMII and MS. Their beneficial effects on cardiovascular complications have been established in clinical and experimental settings. Diet has proven to reduce cardiac hypertrophy, to ameliorate diastolic function, insulin resistance and blood pressure in MS [6-8]. ACE-I improves endothelial function, reverses cardiovascular remodeling effects and has an anti-atherosclerotic effect [9-11]. It remains unclear to what extent these observations can be explained by intrinsic cardiomyocyte dysfunction and/or extrinsic factors influencing cardiomyocyte or cardiac contractility.

The first aim of our study was to phenotype cardiomyocyte contractility and $\mathrm{Ca}^{2+}$ handling in DKO to gain further insight in the contributing mechanisms to cardiac failure in MS. A second goal was to study the effect of in vivo treatment with diet or ACE-I on cardiomyocyte contractility and $\mathrm{Ca}^{2+}$ handling.

\section{Methods}

\section{Animals}

Experiments were conducted in homozygous LDL-receptor (LDLR-/-) and leptin-deficient (ob/ob), double knock-out mice (DKO) of either gender at 12, 18 and 24 weeks with C57BL/6J (WT), LDLR-/- and ob/ob as reference data. Mice were backcrossed for at least 10 generations into C57BL/6J, exhibiting 98.4\% C57BL/6J background. Heterozygous ob/+ and C57BL/6J were purchased from Jackson Laboratory (Bar Harbor, ME, USA). Homozygous ob/ob and DKO mice were generated as described previously [12]. The investigation conforms to the Guide for the Care and Use of Laboratory Animals published by the US National Institutes of Health (NIH Publication No.85-23, revised 1996) and approved by the institutional review board.

\section{Metabolic analysis}

Blood was taken by tail bleeding into EDTA-tubes, after 24 hours fasting.

Serum glucose was measured with a glucometer (Menarini Diagnostics). Plasma was acquired by centrifugation. Triglycerides (TGL), total cholesterol (Chol), highdensity lipoprotein (HDL) and low-density lipoprotein levels (LDL) were determined with a diagnostic reagent kit (Roche).

\section{Food restriction}

Mice were fed standard chow containing 9\% fat (Ssniff, Germany). Food intake of free-fed DKO and LDLR-/mice was respectively 5.7 and $2.5 \mathrm{~g} /$ day [13]. Food intake of diet-restricted DKO and ob/ob was reduced to the daily food intake of LDLR-/- (2.5 g/day) with free access to water, between 12 and 24 weeks of age.

\section{ACE-inhibition}

Captopril $(10 \mathrm{mg} / \mathrm{kg})$ [14] was daily injected intraperitoneally (from 12 till 24 weeks of age) in ob/ob and DKO.

\section{Cell isolation}

Ventricular myocytes from 24 weeks-old mice were isolated as described previously [15]. Briefly, mice were heparinized $(5000 \mathrm{IU} / \mathrm{ml})$ and euthanized with pentobarbital $(50 \mathrm{mg} / \mathrm{ml}$, ip). The heart was excised and weighted. Single left ventricular myocytes were obtained by enzymatic dissociation through retrograde perfusion of the aorta, using collagenase A (0.4-0.5 mg/ml, Roche Diagnostics, Germany) and protease (type XIV 0.08 $\mathrm{mg} / \mathrm{ml}$, Sigma, USA). Cells were used within 5 hours after isolation.

\section{Measurement of cellular contraction, $\left[\mathrm{Ca}^{2+}\right]_{\mathrm{i}}$ transients (CaT) and SR Ca ${ }^{2+}$ content}

Unloaded cell shortening was measured by video-edge detection (Crescent, Salt Lake City, USA) during field stimulation (Myopacer Cell Stimulator, IonOptix, Milton, USA). Cells were stimulated at 1,2 and $4 \mathrm{~Hz}$ to determine a shortening-frequency relationship. Cell contraction is expressed as fractional cell shortening (FCS) i.e. cell length change normalized to resting cell length. Kinetics of contraction were evaluated as time-to-peak contraction (TTP) and relaxation as time to 50\% relaxation (RT50), starting from peak contraction.

$\mathrm{CaT}$ were measured in cardiomyocytes, preloaded with Fluo-3 AM (10 $\mu \mathrm{M}$ for 20 minutes), using perforated patch-clamp and data were calibrated as previously 
described [16]. SR $\mathrm{Ca}^{2+}$ load was estimated as the peak $\mathrm{CaT}$ obtained during $7 \mathrm{~s}$ fast application of $10 \mathrm{mM}$ caffeine. NCX function was evaluated as the decline of the caffeine evoked CaT. In a subset of cells, ruptured patch-clamp was used to asses L-type $\mathrm{Ca}^{2+}$ current $\left(\mathrm{I}_{\mathrm{CaL}}\right)$. The voltageclamp protocol consisted of a pre-step from -70 to -50 $\mathrm{mV}$ to inactivate $\mathrm{I}_{\mathrm{NaL}}$, followed by depolarizing voltage steps from -40 up to $+60 \mathrm{mV}$; duration of the step was $200 \mathrm{~ms}$. Pipette solution was Cs-aspartate based with $10 \mathrm{mM} \mathrm{NaCl}$.

The study on $\mathrm{Ca}^{2+}$ handling was performed in untreated and food-restricted DKO using WT as control.

Response to $\mathrm{Ca}^{2+}$ challenge and $\beta$-adrenergic stimulation Contractile reserve was studied at $1 \mathrm{~Hz}$ by using isoproterenol (50 $\mathrm{nM}$ ) (non-selective $\beta$-adrenoreceptor agonist) or by increasing extracellular $\mathrm{Ca}^{2+}$ (from 1 to
$1.8 \mathrm{mM}$ ). FCS was measured at steady-state and expressed as percent increase in FCS.

All experiments were performed at $35^{\circ} \mathrm{C}$.

\section{Data management and statistical analysis}

Analysis of data was performed using Clampfit 8.2 (Axon Instruments, Scotland), Origin (Originlab, USA) and Ionwizard (IonOptix, USA). Data are expressed as mean \pm standard error of the mean (SEM).' $N$ ' denotes number of animals and ' $n$ ' denotes number of cells. Differences between groups were analyzed for statistical significance by ANOVA with a Fisher post-hoc test, using Statistica 7.1 (StatSoft, USA). $\mathrm{p}<0.05$ was considered significant.

\section{Results}

We did not see any difference between WT and LDLR-/-. Therefore, results obtained from LDLR-/- will not be reported. Additionally, ACE-I did not alter in vivo

Table 1 Metabolic and phenotypic evolution

\begin{tabular}{|c|c|c|c|c|c|c|c|}
\hline \multirow[b]{2}{*}{ At $12 \mathrm{w}$} & \multirow[t]{2}{*}{ WT } & \multicolumn{3}{|c|}{ Ob/ob } & \multicolumn{3}{|c|}{ DKO } \\
\hline & & Untreated & Food restriction & ACE-I & Untreated & Food restriction & ACE-I \\
\hline Body Weight(g) & $21.1 \pm 1.0$ & $49.5 \pm 0.6^{*}$ & $49.8 \pm 0.9^{*}$ & $49.0 \pm 0.3^{*}$ & $48.8 \pm 0.7^{*}$ & $51.2 \pm 0.6^{*}$ & $45.2 \pm 1.6^{*}$ \\
\hline Glycemia(mg/dL) & $56 \pm 2.0$ & $159.3 \pm 18.5^{*}$ & $166 \pm 13.7^{*}$ & $155 \pm 14.5^{*}$ & $155 \pm 10.2^{*}$ & $156 \pm 8.8^{*}$ & $160 \pm 12.9^{*}$ \\
\hline Total Chol(mg/dL) & $104 \pm 25$ & $129 \pm 6^{£}$ & $131 \pm 6$ & $116 \pm 6$ & $977 \pm 45^{*+}$ & $958 \pm 77^{*}$ & $735 \pm 90^{* £}$ \\
\hline Triglycerides $(\mathrm{mg} / \mathrm{dL})$ & $115 \pm 15$ & $53 \pm 2^{* \pm}$ & $53 \pm 2^{*}$ & $69 \pm 3^{*}$ & $585 \pm 66^{*+}$ & $618 \pm 75^{*}$ & $455 \pm 69^{*}$ \\
\hline $\mathrm{HDL}(\mathrm{mg} / \mathrm{dL})$ & $68 \pm 11$ & $106 \pm 5^{* \pm}$ & $107 \pm 5^{*}$ & $90 \pm 4^{*}$ & $232 \pm 5^{*+}$ & $112 \pm 10^{* \pm}$ & $137 \pm 11^{* \pm}$ \\
\hline \multirow[t]{2}{*}{$\mathrm{LDL}(\mathrm{mg} / \mathrm{dL})$} & $16 \pm 11$ & $13 \pm 2$ & $14 \pm 2$ & $12 \pm 3$ & / & / & $308 \pm 228^{*}$ \\
\hline & WT & \multicolumn{3}{|c|}{ Ob/ob } & \multicolumn{3}{|c|}{ DKO } \\
\hline At $18 \mathrm{w}$ & & Untreated & Food restriction & ACE-I & Untreated & Food restriction & ACE-I \\
\hline Body Weight(g) & $22.1 \pm 1.3$ & $56.6 \pm 0.9^{*}{ }^{f}$ & $37.1 \pm 1.1^{*+}$ & $54.0 \pm 0.4^{*}$ & $49.8 \pm 3.4^{*+}$ & $37.3 \pm 0.7^{* \pm}$ & $53.9 \pm 1.8^{*}$ \\
\hline Glycemia(mg/dL) & $72 \pm 4.5$ & $134 \pm 18.9$ & $176 \pm 19.9^{*}$ & $119 \pm 9.1$ & $142 \pm 13.4$ & $158 \pm 19.8^{*}$ & $191 \pm 24.3^{*}$ \\
\hline Total Chol(mg/dL) & $80 \pm 7$ & $154 \pm 13^{*}$ & $77 \pm 6^{+}$ & $140 \pm 10^{*}$ & $579 \pm 145^{*^{+}}$ & $794 \pm 59^{*}$ & $527 \pm 63^{*}$ \\
\hline Triglycerides $(\mathrm{mg} / \mathrm{dL})$ & $75 \pm 6$ & $81 \pm 2$ & $74 \pm 5$ & $70 \pm 2$ & $334 \pm 80^{*+}$ & $331 \pm 38^{*}$ & $171 \pm 19^{ \pm}$ \\
\hline $\mathrm{HDL}(\mathrm{mg} / \mathrm{dL})$ & $62 \pm 6$ & $123 \pm 9^{* E}$ & $67 \pm 7^{+}$ & $112 \pm 7^{*}$ & $100 \pm 6^{*+}$ & $201 \pm 18^{* \pm}$ & $159 \pm 11^{ \pm}$ \\
\hline \multirow[t]{2}{*}{$\underline{\mathrm{LDL}}(\mathrm{mg} / \mathrm{dL})$} & $4 \pm 2$ & $15 \pm 4^{*}$ & I & $16 \pm 3^{*}$ & $277 \pm 100^{*+}$ & $519 \pm 35^{* \pm}$ & $334 \pm 50^{*}$ \\
\hline & WT & \multicolumn{3}{|c|}{$\mathrm{Ob} / \mathrm{ob}$} & \multicolumn{3}{|c|}{ DKO } \\
\hline At $24 \mathrm{w}$ & & Untreated & Food restriction & ACE-I & Untreated & Food restriction & ACE-I \\
\hline Body Weight(g) & $27.1 \pm 0.9$ & $64.5 \pm 0.9^{*}$ & $34.0 \pm 2.3^{*+}$ & $65.8 \pm 1.3^{*}$ & $61.2 \pm 1.7^{*}$ & $35.9 \pm 1.3^{* \pm}$ & $61.8 \pm 1.0^{*}$ \\
\hline Heart Weight(mg) & $164.4 \pm 9.6$ & $196.1 \pm 9.3^{*}$ & $136.4 \pm 2.8^{*+}$ & $178.7 \pm 4.0$ & $184.2 \pm 10.0$ & $132.7 \pm 3.0^{* \pm}$ & $161.2 \pm 4.0^{£}$ \\
\hline HW/BW ratio(mg/g) & $6.2 \pm 0.5$ & $3.1 \pm 0.1^{*}$ & $4.3 \pm 0.3^{*+}$ & $2.7 \pm 0.1^{*}$ & $3.1 \pm 0.1^{*}$ & $3.8 \pm 0.2^{*}$ & $2.6 \pm 0.1^{*}$ \\
\hline Glycemia(mg/dL) & $71 \pm 4,5$ & $166 \pm 11.8^{*}$ & $142 \pm 12.1^{*}$ & $84 \pm 5.0^{+}$ & $141 \pm 28.6^{*}$ & $153 \pm 18.2^{*}$ & $124 \pm 8.7^{*}$ \\
\hline Total Chol(mg/dL) & $93 \pm 17$ & $139 \pm 10$ & $74 \pm 8$ & $145 \pm 10$ & $576 \pm 78^{*+}$ & $816 \pm 74^{* \pm}$ & $634 \pm 55^{*}$ \\
\hline Triglycerides $(\mathrm{mg} / \mathrm{dL})$ & $68 \pm 9$ & $68 \pm 3$ & $69 \pm 5$ & $76 \pm 4$ & $210 \pm 30^{*+}$ & $301 \pm 60^{*}$ & $202 \pm 21^{*}$ \\
\hline $\mathrm{HDL}(\mathrm{mg} / \mathrm{dL})$ & $73 \pm 9$ & $97 \pm 6$ & $66 \pm 8$ & $100 \pm 2$ & $183 \pm 15^{*+}$ & $232 \pm 21^{* \pm}$ & $192 \pm 4^{*}$ \\
\hline $\mathrm{LDL}(\mathrm{mg} / \mathrm{dL})$ & $8 \pm 7$ & $29 \pm 5$ & / & $34 \pm 8$ & $351 \pm 66^{*^{+}}$ & $432 \pm 24^{*}$ & $402 \pm 49^{*}$ \\
\hline
\end{tabular}

This table shows the metabolic and phenotypic evolution from 12 till 24 weeks of age of WT $(\mathrm{N}=13)$, untreated ob/ob $(\mathrm{N}=10)$, untreated DKO $(\mathrm{N}=13)$, food-restricted ob/ob $(N=32)$, food-restricted DKO $(N=19)$, ob/ob treated with ACE-I $(N=14)$ and DKO treated with $A C E-I(N=16)$.

* denotes $\mathrm{p}<0.05$ vs. WT, $+\mathrm{p}<0.05$ vs. untreated ob/ob and $£ \mathrm{p}<0.05$ vs. untreated DKO; HW heart weight, BW body weight, Total Chol total cholesterol, HDL high-density lipoprotein, $L D L$ low-density lipoprotein and / = No data available. 
cardiac contractility in WT [17]. Therefore, cardiomyocyte contractility of WT after ACE-I has not been studied.

\section{Phenotypic and metabolic evolution (Table 1)}

Phenotypic and metabolic features of MS in untreated DKO At 24 weeks, DKO and ob/ob body weight (BW) was significantly higher, compared to WT. Similarly, heart weight $(\mathrm{HW})$ was higher in DKO and ob/ob, resulting in a significantly lower heart weight over body weight ratio (HW/BW ratio), compared to WT. DKO and ob/ob showed a significant hyperglycemia. Additionally, DKO showed a marked atherogenic dyslipidemia (high levels of Chol, TGL and LDL), compared to WT and ob/ob.

\section{Significant weight loss with hypocaloric diet}

Diet resulted in significant weight loss in DKO and ob/ob, associated with significantly lower HW. HW/
BW ratio was significantly increased in ob/ob after diet, compared to untreated ob/ob. Less marked effect of diet on HW/BW ratio was obtained in DKO. Hyperglycemia was reduced in ob/ob, whereas hyperglycemia and atherogenic dyslipidemia were still present in DKO after diet. The same was observed at 12 and 18 weeks.

\section{Significantly lower heart weight in DKO after in vivo treatment with ACE-I}

ACE-I resulted in a significantly lower HW in DKO, but unchanged BW, leading to a small decrease in HW/BW ratio. Neither HW, nor BW were influenced by ACE-I in ob/ob. Fasting glycemia was no longer increased in ob/ob whereas hyperglycemia and atherogenic dyslipidemia remained present in DKO after ACE-I.
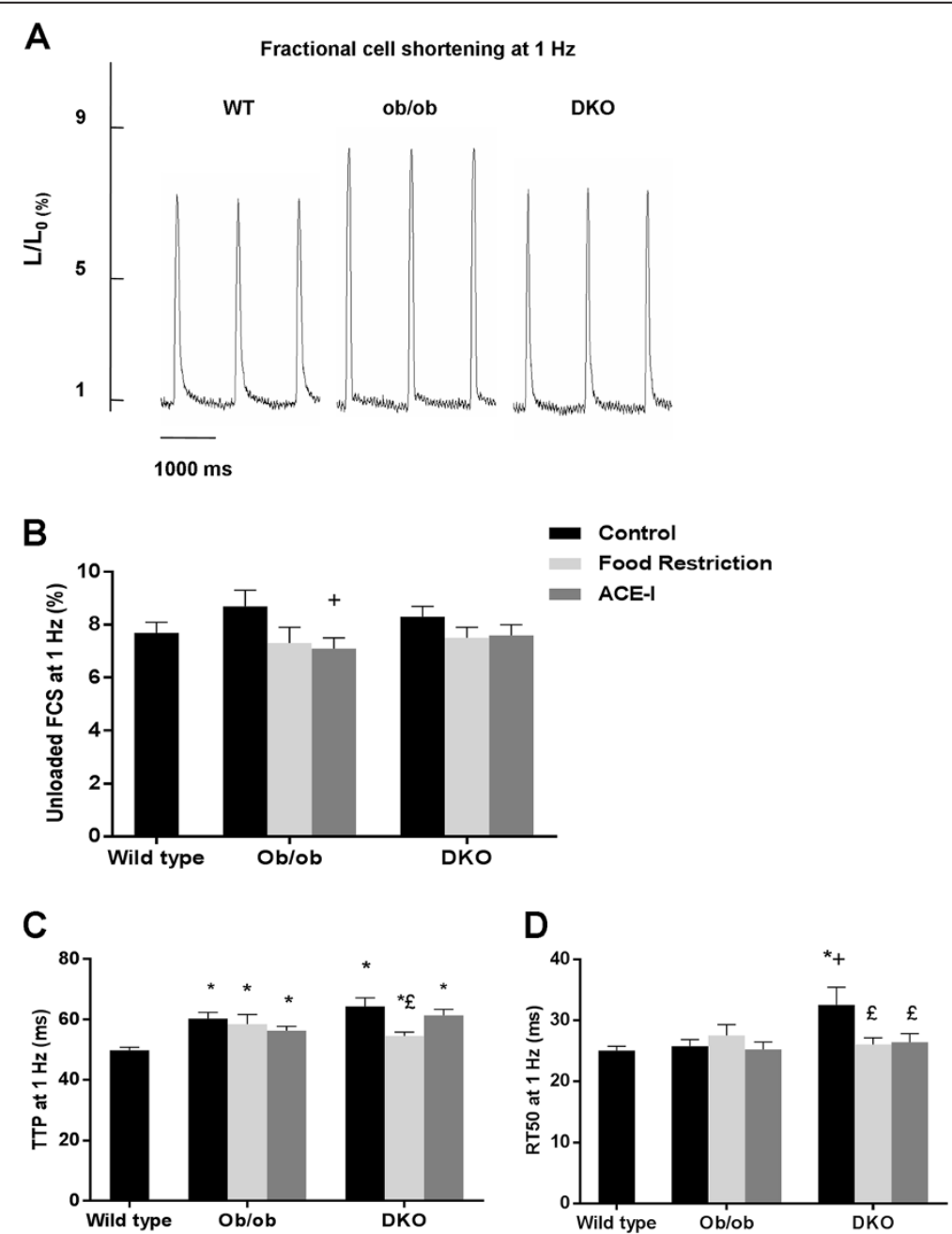

Figure 1 Cell contraction at baseline in WT, ob/ob and DKO at 24 weeks. Representative example of unloaded cell shortening at $1 \mathrm{~Hz}$ in WT, ob/ob and DKO (A), unloaded cell shortening (B), TTP (C) and RT50 (D) at $1 \mathrm{~Hz}$ stimulation frequency in WT $(n=44), o b / o b(n=24)$ and DKO $(n=25) .{ }^{*}$ denotes $p<0.05$ vs. WT, $+p<0.05$ vs. untreated ob/ob and $£ p<0.05$ vs. untreated DKO. 


\section{Cardiomyocyte contractility in vitro Prolongation of excitation-contraction cycle in untreated DKO}

A similar, negative shortening-frequency relationship was observed in DKO, WT and ob/ob (data not shown). Cell width was significantly larger in DKO $(13.4 \pm 0.3$ $\mu \mathrm{m})$ and ob/ob $(13.5 \pm 0.3 \mu \mathrm{m})$ compared to WT $(9.5 \pm 0.2$ $\mu \mathrm{m})$, with similar resting cell length in all groups (data not shown). Figure $1 \mathrm{~A}$ is a representative example of unloaded cardiomyocyte shortening at $1 \mathrm{~Hz}$ in WT, ob/ ob and DKO with similar FCS in all genotypes (Figure 1B). TTP was significantly longer in DKO and ob/ob than in WT (Figure 1C). RT50 was significantly longer in DKO, than in WT and ob/ob (Figure 1D). Regarding contractile reserve, cell shortening increased in all genotypes upon isoproterenol application or increased extracellular $\mathrm{Ca}^{2+}$. However, the relative increase was in both situations smaller in ob/ob and further reduced in DKO, compared to WT (Figure 2).

\section{Normalization of contraction cycle in DKO after diet}

Diet improved contraction and relaxation in DKO only; TTP and RT50 were significantly shorter after diet in DKO, compared to untreated DKO (Figure $1 \mathrm{C}$ and D). However, the response to isoproterenol or increased $\mathrm{Ca}^{2+}$ remained impaired in DKO, as well as in ob/ob (Figure 2).

\section{Partial rescue of contractile reserve after in vivo treatment with ACE-I}

DKO developed a significantly faster relaxation after ACE-I, but TTP was unaffected (Figure $1 C$ and D). Unlike diet, ACE-I leads to a significant increase in $\beta$-adrenergic response in DKO, compared to untreated DKO. Response to extracellular $\mathrm{Ca}^{2+}$ was not improved. In ob/ob, there was a clear tendency towards improvement of the response to isoproterenol or increased $\mathrm{Ca}^{2+}$, without reaching significance (Figure 2).

\section{Calcium handling in isolated cardiomyocytes Impaired $\mathrm{Ca}^{2+}$ handling in untreated DKO}

Figure 3A shows a representative example of $\mathrm{CaT}$ obtained during short depolarizing pulses at $1 \mathrm{~Hz}$ in WT and DKO. Diastolic $\mathrm{Ca}^{2+}$ at 0.5, 1, 2 and $4 \mathrm{~Hz}$ was not different between WT and DKO (data not shown). At 1 and $4 \mathrm{~Hz}$, CaT amplitude in DKO was significantly smaller (Figure 3B), associated with slower kinetics (longer TTP and slower relaxation) (Figure $3 \mathrm{C}$ and $\mathrm{D}$ ), compared to WT. SR $\mathrm{Ca}^{2+}$ content, evaluated as the peak CaT evoked during caffeine application was not different between DKO and WT (Figure 4A). Decline of the caffeine evoked $\mathrm{CaT}$ was prolonged in DKO compared to WT (Figure 4B). $\mathrm{I}_{\mathrm{CaL}}$ density and voltage-dependence were similar for WT and DKO (Figure 4C).

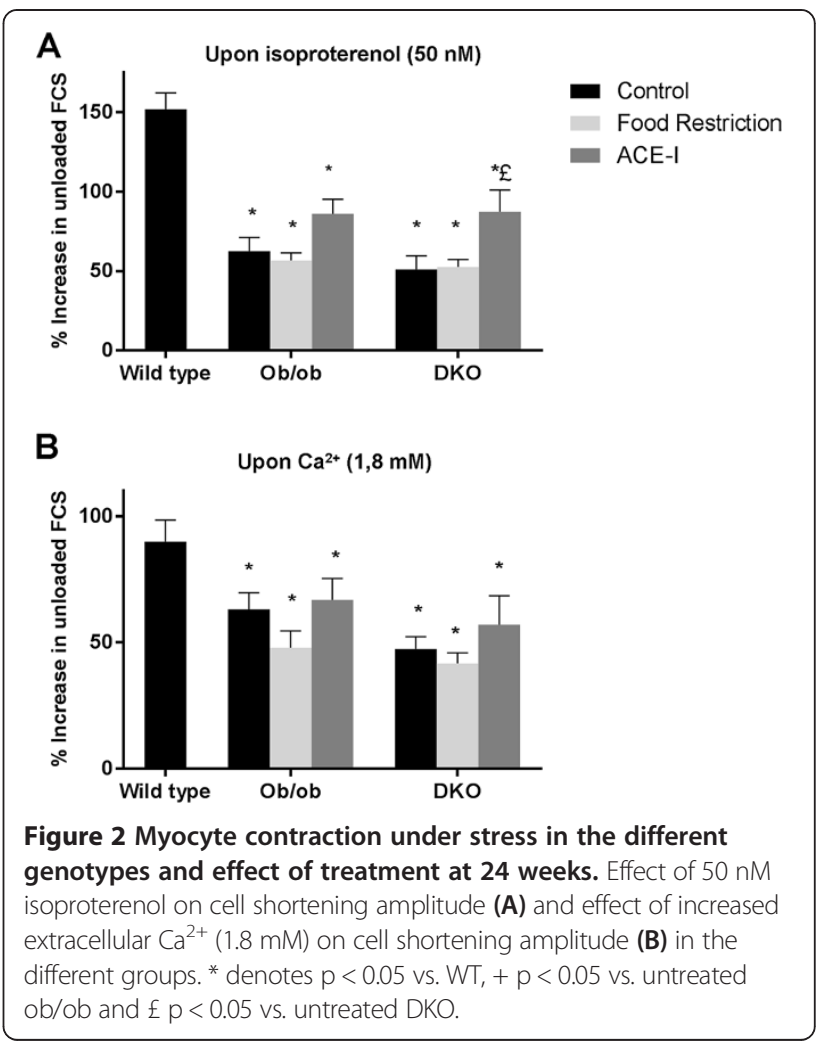

Improved $\mathrm{Ca}^{2+}$ handling in DKO after diet

Diet resulted in a significant increase in CaT amplitude in DKO with faster kinetics, in particular TTP (Figure 3). $\mathrm{SR} \mathrm{Ca}^{2+}$ content remained comparable between the groups after diet (Figure 4A). $\mathrm{Ca}^{2+}$ decline during caffeine application was improved with diet (Figure 4B). $\mathrm{I}_{\mathrm{CaL}}$ remained unchanged after diet (Figure 4C).

\section{Discussion}

In this study, we determined cardiomyocyte contractility and $\mathrm{Ca}^{2+}$ handling in isolated cardiomyocytes in a mouse model of MS. The first new finding of our study is functional characterization of single cardiomyocytes in a mouse model of MS. At the cellular level, MS is characterized by slower kinetics of contraction and relaxation. DKO mice show a significantly longer TTP and RT50, indicating prolongation of contraction-relaxation cycle. The second finding is that contractile reserve is impaired in isolated cardiomyocytes from MS-mice. ACE-I, but not weight reduction, can restore the response to $\beta$-adrenergic stimulation. However, the attenuated response to extracellular $\mathrm{Ca}^{2+}$ is not improved by the aforementioned treatments.

Both findings are a hallmark of failing myocardium. Furthermore, these cellular defects are in parallel with previous in vivo studies in our laboratory and with clinical studies $[1,2,6-8]$. 

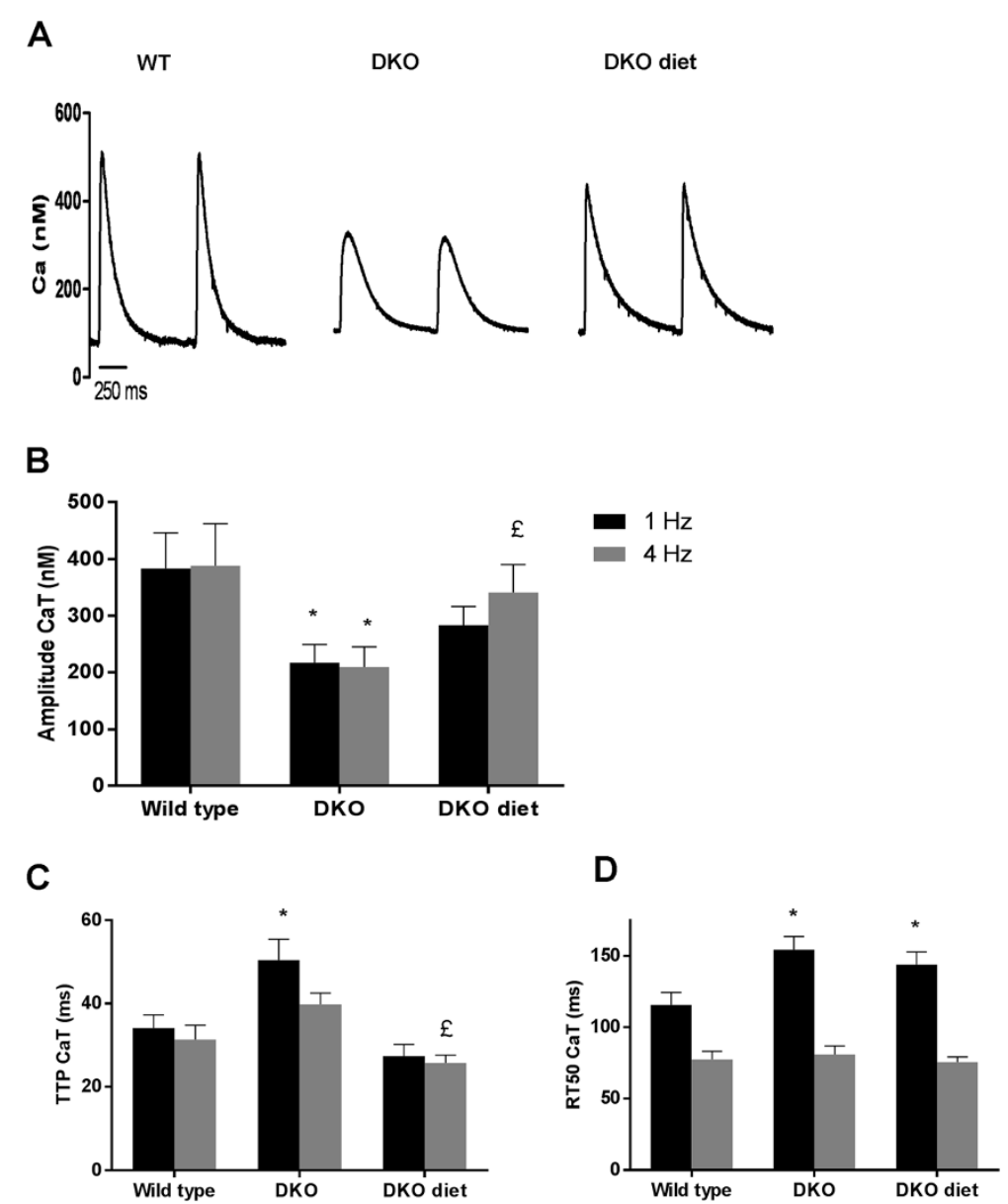

Figure 3 CaT properties in WT, DKO and DKO undergoing hypocaloric diet. (A) Representative example of CaT recording during short depolarizing pulses at $1 \mathrm{~Hz}$ in WT, DKO and DKO after diet. CaT amplitude (B) time to peak (C) and RT50 (D) in WT $(n=14)$, DKO $(n=21)$ and DKO diet $(n=18) .{ }^{*}$ denotes $p<0.05$ vs. WT and $£ p<0.05$ vs. untreated DKO.

\section{Diastolic dysfunction}

In vivo, diastolic dysfunction in MS is characterized by an early and late component. The late component is due to enhanced myocardial fibrosis, resulting in decreased end-diastolic volume [2]. The early component is characterized by impaired active relaxation. Early relaxation occurs when $\mathrm{Ca}^{2+}$ is removed from the cytoplasm into the SR via SERCA2a and out of the cell by the NCX, and reflects dissociation of $\mathrm{Ca}^{2+}$ from contractile myofilaments. SERCA2a is a membrane protein that catalyzes ATPdependent transport of $\mathrm{Ca}^{2+}$ from the cytosol into the SR and regulated by PLB. In 24 weeks-old DKO, preserved $\mathrm{PLB} / \mathrm{SERCA2a}$ ratio is associated with decreased phosphorylation of PLB, resulting in decreased activity of SERCA2a [2]. NCX is considered as the second most important mechanism for removing $\mathrm{Ca}^{2+}$ from the cytosol. Hattori et al. showed impaired NCX function in type I DM (DMI), due to decreased protein expression, playing an important role in altered $\mathrm{Ca}^{2+}$ handling [18]. In DKO, we report a significantly longer RT50 for cardiomyocyte contraction and $\mathrm{CaT}$, mainly related to SERCA2a and PLB. $\mathrm{Ca}^{2+}$ decline after administration of caffeine is significantly prolonged in DKO, indicating altered NCX function. Although, we did not look at changes in NCX expression, it is likely that both mechanisms (decreased phosphorylation of PLB and altered NCX function) contribute to impaired relaxation in MS. Finally, unaltered levels of resting calcium indicate that the "pump-leak balance" is unchanged, as previously described in DMI [4].

After in vivo treatment with diet and ACE-I, RT50 is comparable with WT. Diet also improved $\mathrm{Ca}^{2+}$ handling in DKO. These findings are consistent with previous in vivo studies, showing diet to improve cardiac function and insulin sensitivity in MS-mice [5-7]. The beneficial effect of ACE-I is largely due to improved endothelial function, its anti-atherosclerotic and anti-remodeling effects [9-11,19]. All together, our data provide evidence for contribution of intrinsic cellular mechanisms, in recovering diastolic function after diet and ACE-I, 


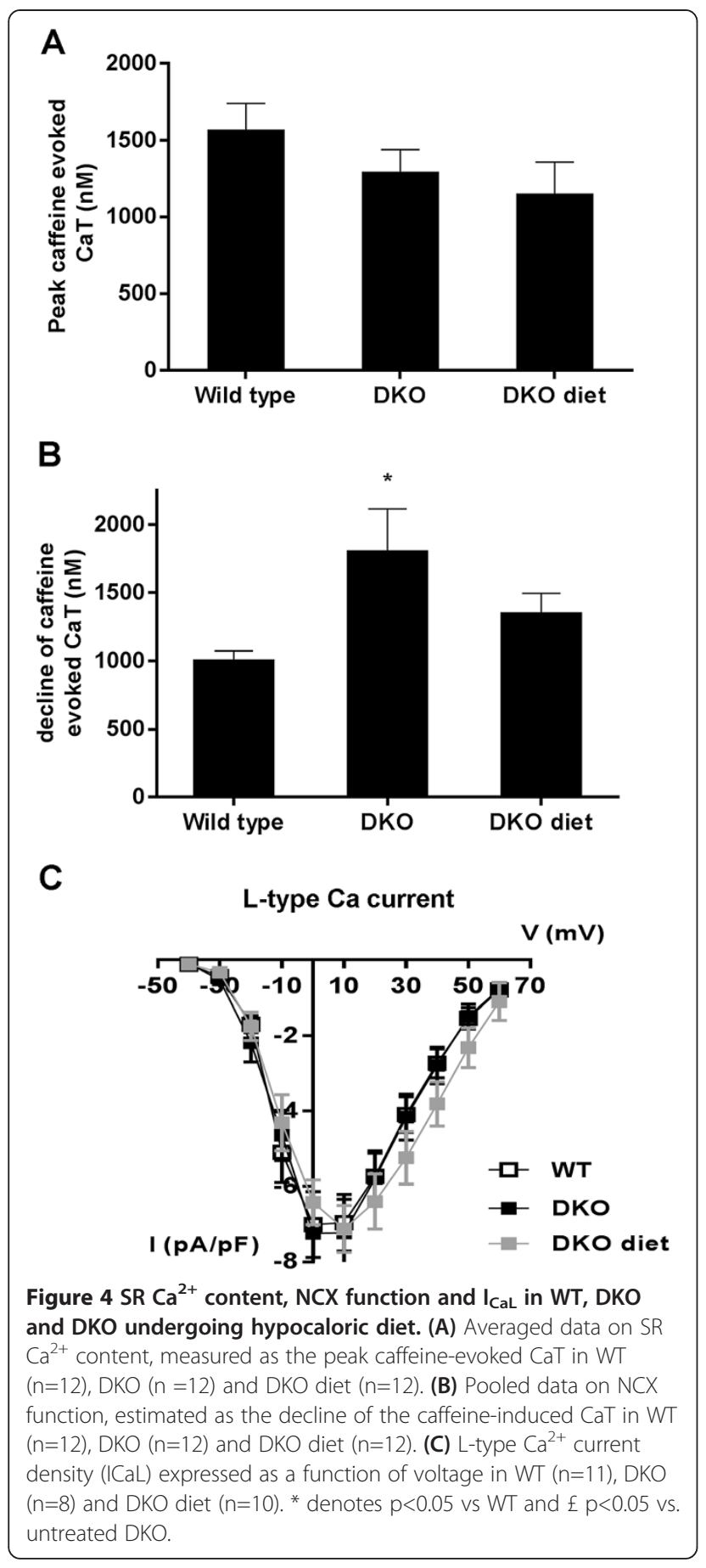

associated with extrinsic mechanisms (eg. reduced ventricular-vascular stiffening, reduced subendothelial lipid deposition and increased endothelium-dependent relaxation) [6-11,19].

\section{Systolic dysfunction}

In vivo, MS is characterized by systolic dysfunction, reflected by reduced cardiac output [2]. Cellular contraction starts when $\mathrm{Ca}^{2+}$ enters the cell via $\mathrm{L}$-type $\mathrm{Ca}^{2+}$ channels and thereby activating RyR that will lead to further $\mathrm{Ca}^{2+}$ release from the SR. $\mathrm{Ca}^{2+}$ will in turn activate myofilaments, inducing contraction. This process is known as calcium-induced-calcium-release, playing a key role in cellular contraction.

In DMI, L-type $\mathrm{Ca}^{2+}$ channel activity is unchanged, but RyR protein levels are decreased [4]. DKO have a significantly smaller CaT amplitude and a significantly larger TTP. These data, taken together with lower protein levels of RyR, suggest RyR to be likely responsible for the slow $\mathrm{Ca}^{2+}$ release from the SR, since no changes in L-type $\mathrm{Ca}^{2+}$ current were found. Our data in DKO show that FCS is preserved despite a decrease in CaT amplitude. This might be explained by an increase in myofilament $\mathrm{Ca}^{2+}$ sensitivity. However, we did not asses myofilament sensitivity in DKO and experiments on contractility and $\mathrm{Ca}^{2+}$ handling were conducted in different cell groups. It is also unclear how DM affects myofilament sensitivity. Whereas some studies suggested that DM is associated with decreased myofilament sensitivity, others indicate the opposite [20,21]. On the other hand, it is important to notice that slowed relaxation, as found in DKO, is also a feature of increased myofilament sensitivity. Diet and ACE-I did not influence TTP of cellular contraction. In contrast, diet did improve kinetics of CaT in DKO, in particular TTP, suggesting that diet might influence expression or activity of RyR or myofilament sensitivity.

\section{Decreased $\beta$-responsiveness}

Depressed $\beta$-responsiveness is a hallmark of heart failure. The mechanisms underlying impaired $\beta$-responsiveness have been extensively studied. It remains unclear whether this attenuated response is attributable to decreased expression of $\beta$-receptors, to distal transduction pathways defects and/or to impaired $\mathrm{Ca}^{2+}$ handling. Previous studies showed a decreased $\beta$-receptor expression/ activity; however, more recent studies demonstrate impaired $\beta$-inotropy, without changes in $\beta$-adrenoceptor expression/activity [22,23]. Minhas et al. showed impaired $\beta$-adrenergic response in ob/ob, due to altered PKA-activity and reversible by leptin repletion [24]. We confirmed impaired contractile reserve in ob/ob and found a more pronounced impairment in DKO. Although we did not study $\beta$-receptor expression/activity or distal signaling pathways, it is likely that altered PKA-activity might also play a role in DKO, due to the same lack of leptin in these mice. Another possible contributor is reduced insulin sensitivity. As shown, DKO are hyperglycemic, hyperinsulinemic and insulin resistant. Insulin might contribute to cardiomyocyte $\beta$-responsiveness because of cross-talk between $\beta$-adrenergic and insulin receptors, mediated via Akt-pathway [25]. Consistent with this cross-talk, overexpression of insulin growth factor improves $\beta$-response in DMI [26]. On the other 
hand, insulin treatment in animals with DMI did not alter PKA-mediated activity and phosphorylation [27]. Whether insulin resistance contributes to $\beta$-inotropic hyporesponsiveness remains controversial. Yonemochi et al. showed in cultured ventricular neonatal rat myocytes that captopril enhances $\beta$-adrenergic responsiveness by inducing $\beta$-adrenergic receptor upregulation, mediated via activation of bradykinin B2-receptors and PKC [28].

Our study gives more insight in the pathophysiological mechanisms contributing to heart failure in MS and provides potential therapeutic targets in prevention and treatment of cardiovascular complications in MS.

\section{Limitations of our study}

A limitation of our study is leptin deficiency in our mice. In humans, obesity is associated with hyperleptinemia. Long-term hyperleptinemia results in leptin resistance. Leptin resistance and interrupted leptin signaling were reported in cardiomyocytes under hyperleptinemic conditions, making hyperleptinemia comparable to leptin deficiency regarding signaling pathways of leptin [29].

A possible contributor to our results after diet is the extreme weight loss that our mice experienced. It was remarkable that heart weight in DKO and ob/ob after diet was lower, compared to WT. Extreme weight loss eg. starvation, has detrimental effects on cardiac performance [30]. These adverse starvation-effects strongly emphasize the need for tightly controlled weight reduction.

In the present study, we did not study the effect of ACE-I or $\beta$-adrenergic stimulation on $\mathrm{Ca}^{2+}$ handling, neither did we investigate molecular changes in calcium handling or contractile proteins. These remain interesting issues for future research.

\section{Conclusion}

In vivo cardiac dysfunction, as seen in MS and shown previously in our laboratory and in humans, can at least partially be explained by alterations of intrinsic properties of cardiomyocytes $[1,2,8,9]$. We have shown that cardiomyocyte contractility and $\beta$-adrenergic response are impaired in MS, due to alterations in $\mathrm{Ca}^{2+}$ handling. ACE-I, but not weight loss, can restore impaired cardiomyocyte response to $\beta$-adrenergic stimulation in MS-mice.

\footnotetext{
Abbreviations

ACE-I: Angiotensin-converting-enzyme inhibition; CaT: Calcium transient; DKO: Double knock-out mouse; DMI: Diabetes Mellitus type I; DMIl: Diabetes Mellitus type II; FCS: Fractional cell shortening; LDLR-/-: Low-density lipoprotein receptor knock-out mouse; NCX: Sodium-calcium exchanger; Ob/ob: Leptin-deficient mouse; PLB: Phospholamban; RT50: Time to 50\% relaxation; RyR: Ryanodin receptor; SERCA2a: Sarcoplasmatic $\mathrm{Ca}^{2+}$ ATP-ase; SR: Sarcoplasmatic reticulum; TTP: Time-to-peak contraction; WT: Wild type mouse.
}

\section{Competing interests}

The authors declare that they have no competing interests.

\section{Authors' contributions}

IN participated in the conception and design of the study, data acquisition, (statistical) analysis and interpretation of the results and drafting of the manuscript. IN obtained funding for this study (see Acknowledgements). VB participated in acquisition and analysis of data and was involved in drafting the manuscript. GVDM, AVDP, AVDB helped with acquisition and

interpretation of data on phenotypic and metabolic characteristics, as well as the effect of treatment on these chraracteristics. GVDM, AVDP, AVDB critically revised the manuscript. KM, KRS and PH participated in the conception and design of the study. KM and KRS gave a critical revision of the manuscript for important intellectual content. PH participated in the conception and design of the study and in statistical analysis of the data. PH obtained funding for this project (see Acknowledgements) and was involved in drafting and finalizing the manuscript. All authors have read and approved the final manuscript.

\section{Acknowledgements}

This study was supported by a PhD fellowship of the FWO, the Flanders Fund for Scientific Research, (to I.Nevelsteen) and by a grant of the Research Fund KU Leuven - Bijzonder Onderzoeksfonds (OT 04/39, OT 05/55, PF/10/014).

\section{Author details}

${ }^{1}$ Department of Cardiovascular Sciences, Research Unit of Experimental Cardiac Surgery, KU Leuven, Herestraat 49, Leuven B-3000, Belgium. ${ }^{2}$ Research Unit of Experimental Cardiology, KU Leuven, Leuven, Belgium.

Received: 3 March 2013 Accepted: 10 July 2013

Published: 12 July 2013

\section{References}

1. Van den Bergh A, Flameng W, Herijgers P: Type II diabetic mice exhibit contractile dysfunction but maintain cardiac output by favourable loading conditions. Eur J Heart Fail 2006, 8(8):777-783.

2. Van den Bergh A, Vanderper A, Vangheluwe $P$, et al: Dyslipidaemia in type II diabetic mice does not aggravate contractile impairment but increases ventricular stiffness. Cardiovasc Res 2008, 77:371-379.

3. Belke DD, Swanson EA, Dillmann WH: Decreased sarcoplasmic reticulum activity and contractility in diabetic db/db mouse heart. Diabetes 2004, 53(12):3201-3208.

4. Choi KM, Zhong $\mathrm{Y}$, Hoit $\mathrm{BD}$, et al: Defective intracellular $\mathrm{Ca}(2+)$ signaling contributes to cardiomyopathy in Type 1 diabetic rats. Am J Physiol Heart Circ Physiol 2002, 283(4):H1398-H1408.

5. Kranias EG, Bers DM: Calcium and cardiomyopathies. Subcell Biochem 2007, 45:523-537.

6. Van den Bergh A, Vangheluwe $P$, Vanderper A, et al: Food-restriction in obese dyslipidaemic diabetic mice partially restores basal contractility but not contractile reserve. Eur J Heart Fail 2009, 11(12):1118-1125.

7. Alpert MA, Terry BE, Mulekar M, et al: Cardiac morphology and left ventricular function in normotensive morbidly obese patients with and without congestive heart failure, and effect of weight loss. Am J Cardiol 1997, 80(6):736-740.

8. Wong CY, Byrne NM, O'Moore-Sullivan T, Hills AP, Prins JB, Marwick TH: Effect of weight loss due to lifestyle intervention on subclinical cardiovascular dysfunction in obesity (body mass index $>30 \mathrm{~kg} / \mathrm{m} 2$ ). Am J Cardiol 2006, 98(12):1593-1598.

9. Virdis A, Ghiadoni L, Taddei S: Effects of antihypertensive treatment on endothelial function. Curr Hypertens Rep 2011, 13(4):276-281.

10. Pfeffer JM, Pfeffer MA: Angiotensin converting enzyme inhibition and ventricular remodeling in heart failure. Am J Med 1988, 84(3A):37-44.

11. Fennessy PA, Campbell JH, Mendelsohn FA, Campbell GR: Angiotensinconverting enzyme inhibitors and atherosclerosis: relevance of animal models to human disease. Clin Exp Pharmacol Physiol 1996, 23(8):S30-S32.

12. Mertens A, Verhamme P, Bielicki JK, et al: Increased low-density lipoprotein oxidation and impaired high-density lipoprotein antioxidant defense are associated with increased macrophage homing and atherosclerosis in dyslipidemic obese mice: LCAT gene transfer decreases atherosclerosis. Circulation 2003, 107(12):1640-1646.

13. Verreth W, De Keyzer D, Pelat $M$, et al: Weight-loss-associated induction of peroxisome proliferator-activated receptor-alpha and peroxisome proliferator-activated receptor-gamma correlate with reduced 
atherosclerosis and improved cardiovascular function in obese insulin-resistant mice. Circulation 2004, 110(20):3259-3269.

14. Wichi RB, Farah V, Chen Y, Irigoyen MC, Morris M: Deficiency in angiotensin AT1a receptors prevents diabetes-induced hypertension. Am J Physiol Regul Integr Comp Physiol 2007, 292(3):R1184-R1189.

15. Antoons G, Mubagwa K, Nevelsteen I, Sipido KR: Mechanisms underlying the frequency dependence of contraction and $[\mathrm{Ca}(2+)](\mathrm{i})$ transients in mouse ventricular myocytes. J Physiol 2002, 543(Pt 3):889-898.

16. Trafford AW, Díaz ME, Eisner DA: A novel, rapid and reversible method to measure $\mathrm{Ca}$ buffering and time-course of total sarcoplasmic reticulum Ca content in cardiac ventricular myocytes. Pflugers Arch 1999, 437(3):501-503.

17. Van der Mieren G, Nevelsteen I, Vanderper A, Oosterlinck W, Flameng W, Herijgers $P$ : Angiotensin-converting enzyme inhibition and food restriction in diabetic mice do not correct the increased sensitivity for ischemia-reperfusion injury. Cardiovasc Diabetol 2012, 11:89.

18. Hattori Y, Matsuda N, Kimura J, et al: Diminished function and expression of the cardiac $\mathrm{Na}+-\mathrm{Ca} 2+$ exchanger in diabetic rats: implication in Ca2+ overload. J Physiol 2000, 527(Pt 1):85-94

19. Asmar RG, Pannier B, Santoni JP, et al: Reversion of cardiac hypertrophy and reduced arterial compliance after converting enzyme inhibition in essential hypertension. Circulation 1988, 78(4):941-950.

20. Jweied EE, McKinney RD, Walker LA, et al: Depressed cardiac myofilament function in human diabetes mellitus. Am J Physiol Heart Circ Physiol 2005, 289(6):H2478-H2483.

21. Falcão-Pires I, Palladini G, Gonçalves N, et al: Distinct mechanisms for diastolic dysfunction in diabetes mellitus and chronic pressure-overload. Basic Res Cardiol 2011, 106(5):801-814.

22. Strassheim D, Houslay MD, Milligan G: Regulation of cardiac adenylate cyclase activity in rodent models of obesity. Biochem J 1992, 283(Pt 1):203-208.

23. Carroll JF, Kyser CK, Martin MM: Beta-Adrenoceptor density and adenylyl cyclase activity in obese rabbit hearts. Int I Obes Relat Metab Disord 2002, 26(5):627-632.

24. Minhas KM, Khan SA, Raju SV, et al: Leptin repletion restores depressed \{beta\}-adrenergic contractility in ob/ob mice independently of cardiac hypertrophy. J Physiol 2005, 565(Pt 2):463-474. Erratum in: J Physiol. 2005;566(Pt 3):999. Saliaris, Anastasies P.

25. Morisco C, Condorelli G, Trimarco V, et al: Akt mediates the cross-talk between beta-adrenergic and insulin receptors in neonatal cardiomyocytes. Circ Res 2005, 96(2):180-188.

26. Norby FL, Aberle NS 2nd, Kajstura J, Anversa P, Ren J: Transgenic overexpression of insulin-like growth factor I prevents streptozotocin-induced cardiac contractile dysfunction and beta-adrenergic response in ventricular myocytes. J Endocrinol 2004 180(1):175-182.

27. Netticadan T, Temsah RM, Kent A, Elimban V, Dhalla NS: Depressed levels of $\mathrm{Ca} 2+-$ cycling proteins may underlie sarcoplasmic reticulum dysfunction in the diabetic heart. Diabetes 2001, 50(9):2133-2138.

28. Yonemochi H, Yasunaga S, Teshima Y, et al: Mechanism of beta-adrenergic receptor upregulation induced by ACE inhibition in cultured neonatal rat cardiac myocytes: roles of bradykinin and protein kinase C. Circulation 1998, 97(22):2268-2273.

29. Ren J: Leptin and hyperleptinemia - from friend to foe for cardiovascular function. J Endocrinol 2004, 181(1):1-10

30. Poirier P, Eckel RH: Obesity and cardiovascular disease. Curr Atheroscler Rep 2002, 4(6):448-453.

doi:10.1186/1471-2261-13-51

Cite this article as: Nevelsteen et al: ACE-inhibition, but not weight reduction restores cardiomyocyte response to $\beta$-adrenergic stimulation in the metabolic syndrome. BMC Cardiovascular Disorders 2013 13:51.

\section{Submit your next manuscript to BioMed Central and take full advantage of:}

- Convenient online submission

- Thorough peer review

- No space constraints or color figure charges

- Immediate publication on acceptance

- Inclusion in PubMed, CAS, Scopus and Google Scholar

- Research which is freely available for redistribution

Submit your manuscript at www.biomedcentral.com/submit
C Biomed Central 